\title{
Liver Metastasis of Urothelial Carcinoma with Hepatoid Features: An Unusual Morphological Finding
}

\author{
PAUL FRIEDMAN and JIN-PING LAI \\ Department of Pathology, Saint Louis University School of Medicine, Saint Louis, MO, U.S.A.
}

\begin{abstract}
Bladder cancer comprises of many well-known variants and divergent histologies, both of urothelial and adenocarcinomatous type. Extrahepatic tumors with features that resemble hepatocellular carcinoma, often referred to as hepatoid adenocarcinomas, have been described in many different organs, and are aggressive and prognostically unfavorable. Hepatoid features of bladder cancer are unusual and rarely reported. We report a unique case of a metastatic lesion to the liver consisting of a urothelial carcinoma with hepatoid features, a tumor that likely falls within the hepatoid adenocarcinoma spectrum. It is an unusual morphological finding in urothelial carcinoma and is important to recognize due to its potential for aggressive behavior, particularly when it involves a liver lesion in which hepatocellular carcinoma may be suspected.
\end{abstract}

Urothelial carcinoma is the most common type of bladder cancer and has many well-known examples of variant histology and divergent differentiation. The recent World Health Organization classification of urothelial cancer describes 13 histological variants (1). Adenocarcinoma of the bladder is the third most common bladder cancer (after urothelial carcinoma and squamous cell carcinoma), and can be histologically classified as enteric, adenocarcinoma not otherwise specified, mucinous, signet ring cell, clear cell, hepatoid, or mixed (2). Specifically, hepatoid features in bladder cancer are rarely reported. We aimed to bring awareness of this unusual morphological feature by presenting a rather unique case of a metastatic urothelial carcinoma with hepatoid features involving the liver.

Correspondence to: Paul Friedman, MD, Department of Pathology, Saint Louis University School of Medicine, 1402 S. Grand Blvd., St Louis, MO 63104, U.S.A. Tel: +1 3145778782, Fax: +1 3145776132, e-mail: friedmanp@slu.edu

Key Words: Liver metastasis, urothelial carcinoma.

\section{Case Report}

A 71-year-old man presented to the hospital with complaints of dizziness, fever, back pain, fatigue, decreased appetite, and unintentional weight loss of $7 \mathrm{~kg}$ in the previous 3 months. On physical examination, he appeared jaundiced and was noted to have scleral icterus. Past medical and surgical history was significant for hypertension, gastric ulcer, and urothelial carcinoma status post radical cystoprostatectomy 8 years prior. He was also found to have acute kidney injury with a creatinine level of $2.6 \mathrm{mg} / \mathrm{dl}$. Additional pertinent laboratory values include alkaline phosphatase of 387 units/l, aspartate aminotransferase of 223 Units/l, alanine aminotransferase of 167 Units/l, total bilirubin of $6.9 \mathrm{mg} / \mathrm{dl}$, and serum sodium level of $131 \mathrm{mmol} / \mathrm{l}$. Computed tomographic imaging of the abdomen and pelvis revealed an $8.2 \times 4.2 \mathrm{~cm}$ left pelvic mass at the prior surgical site which were of concern for recurrent malignancy, as well as multiple hypoattenuating hepatic lesions which were of concern for metastatic disease. There was also suggestion of liver cirrhosis, therefore primary liver malignancy was also considered.

After discharge from the hospital, the patient underwent outpatient positron-emission tomographic scan, which revealed an 18-fluoro-deoxyglucose (FDG)-avid soft tissue lesion at the left side of the urinary bladder fossa, multiple mediastinal, mesenteric, retroperitoneal and pelvic FDG-avid lymph nodes, and additional metastatic disease involving the liver, left superior pubic ramus, left acetabulum, and tail of the pancreas. He was subsequently scheduled for a liver biopsy.

The cystoprostatectomy specimen obtained 8 years prior had revealed a $2.6 \times 1.5 \mathrm{~cm}$ lesion involving the anterior wall of the urinary bladder. Histologically, it consisted of a highgrade papillary urothelial carcinoma with tumor cells exhibiting features of pleomorphism, increased nuclear to cytoplasmic ratio and mitotic activity, and irregular nuclear membranes. The tumor was invasive into the lamina propria with no involvement of the muscularis propria identified. No lymphovascular invasion or lymph node metastasis were 
identified, and all surgical resection margins were negative for invasive or in situ carcinoma. No specific morphological pattern or divergent differentiation was appreciated.

The liver biopsy demonstrated carcinoma cells arranged in a predominantly trabecular and cord-like pattern. Few foci of glandular differentiation were also noted. The tumor cells had pleomorphic nuclei and with foci that had high nuclear to cytoplasmic ratio. There were also hepatoid-like areas that contained large, polygonal cells with round nuclei and eosinophilic, granular or clear cytoplasm. There were occasional mitoses. Immunohistochemically, the tumor was positive for cytokeratin 7 (CK7) and p63, focally positive for glypican 3, and negative for CK20, synaptophysin, chromogranin, cluster of differentiation 56 (CD56), caudalrelated homeobox gene 2 (CDX2), and thyroid transcription factor 1. A periodic acid-Schiff with diastase (PASD) special stain highlighted rare intracytoplasmic hyaline globules, further evidence to support a hepatoid differentiation (Figure 1). Based on the patient's past clinical history, as well as morphological and immunohistochemical staining profile, this was felt to be most consistent with a metastatic urothelial carcinoma with hepatoid features and focal glandular differentiation. Morphological and immunohistochemical comparison between the present case and an unrelated case of hepatocellular carcinoma is depicted in Figure 2.

Unfortunately, the patient experienced a rapid progression of his urothelial cancer, and due to hepatic and renal failure, he was not a candidate for chemotherapy, and was referred to palliative care where he elected for arrangement of hospice care at home.

\section{Discussion}

Given the morphological and immunohistochemical staining profile, as well as history of a high-grade papillary urothelial carcinoma, we favored this to be a urothelial carcinoma with hepatoid features and focal glandular differentiation rather than a pure adenocarcinoma. To our knowledge, there are no other described cases of urothelial carcinoma with hepatoid features or differentiation. Rather, there are few reported cases of hepatoid adenocarcinoma (HAC) of the urinary bladder, renal pelvis, and ureter.

Since 1994, there have been nine reported cases of HAC of the urinary bladder (3-8). HAC is a rare tumor that is described as being morphologically reminiscent of a hepatocellular carcinoma, including nests and trabecular structures of polygonal atypical cells with abundant granular cytoplasm. Metzgeroth et. al evaluated 262 cases of HAC published in the English literature (9). At the time of publication of the report in 2010, seven $(2.7 \%)$ of these tumors had arisen from the urinary bladder. Overall, this report demonstrated that there was a male predominance and a median age of 65 years at the time of diagnosis. One-year

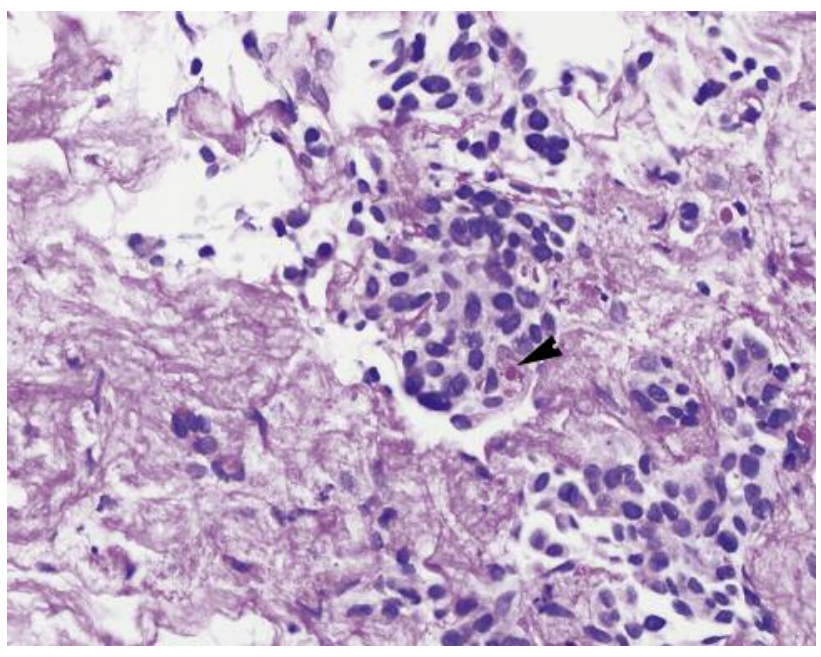

Figure 1. Urothelial carcinoma with periodic acid-Schiff with diastasepositive globule (arrowhead) supporting hepatoid differentiation $(\times 600)$.

survival was 55\% and median overall survival 11 months. A total of $88 \%$ of cases demonstrated alpha-fetoprotein (AFP) positivity by immunohistochemistry (IHC).

HAC is an aggressive neoplasm with an unfavorable prognosis and a high proportion of metastases, with the liver and lymph nodes being common metastatic sites. These tumors are often associated with AFP production, leading to high serum AFP levels and IHC positivity on histological examination of the tissue. In the present case, AFP by IHC was negative. No serum AFP levels were available for review.

Tumors with HAC differentiation have also been described in the renal pelvis or ureter. Ishikura et al. described a tumor of the renal pelvis as a pure adenocarcinoma (HAC) that was AFP-positive by IHC (10). Another example of an AFP-positive renal pelvis tumor was reported by Samaratunga et al. in which there were components of both hepatoid and urothelial differentiation, all of which was positive for AFP (11). Sakata et al. described a renal pelvis mass that also contained both hepatoid and urothelial differentiation, however, only the hepatoid component was positive for AFP (12). Our current case consisted of a urothelial carcinoma with hepatoid and focal glandular features that was AFP-negative. While we favored classifying this tumor as a urothelial carcinoma with hepatoid features and focal glandular differentiation, and other reported bladder and ureter lesions were designated as HAC, we believe this may represent a spectrum of the same disease given the morphological and immunohistochemical heterogeneity of the tumors.

Previous reports of HACs show them to have unfavorable prognoses. The present case is similar, with development of 


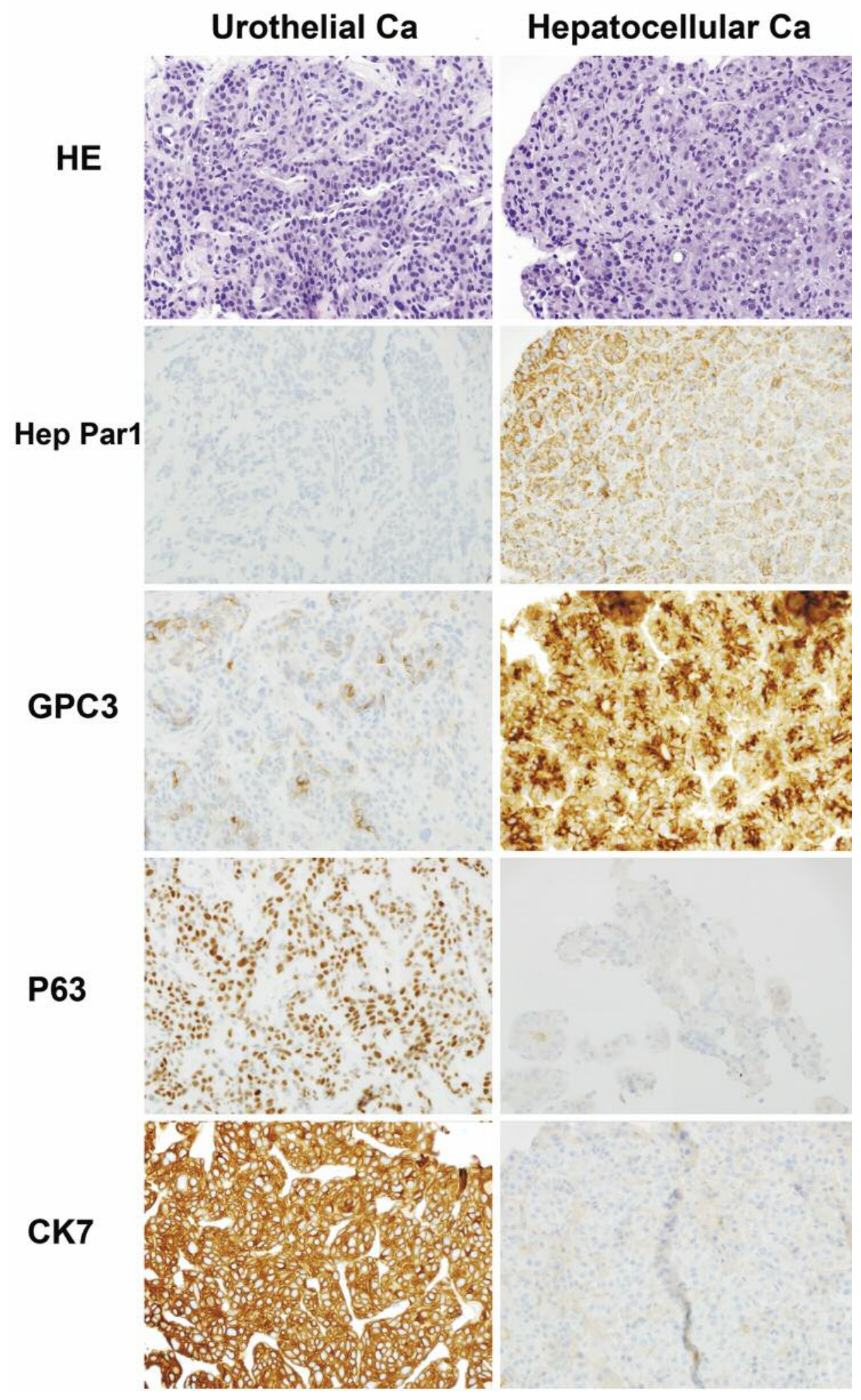

Figure 2. Comparison of urothelial carcinoma with hepatoid features and hepatocellular carcinoma. Hematoxylin and eosin stain shows urothelial carcinoma with round nuclei and abundant eosinophilic cytoplasm. By immunohistochemistry, urothelial carcinoma is hepatocyte specific antigen (Hep Par1)-negative, focally positive for glypican-3 (GPC3), p63-positive, and cytokeratin 7 (CK7)-positive. In contrast, hepatocellular carcinoma is Hep Par1-positive, glypican-3-positive, p63-negative, and cytokeratin 7-negative $(\times 400)$. 
widespread metastases to multiple sites, including the liver, leading to hepatic failure. A hepatoid variant or hepatoid-like features may suggest aggressive behavior and poor prognosis. One specific well-known histological variant in which this is the case is micropapillary urothelial carcinoma, where there is a higher incidence of lymph node metastasis and increased risk of mortality (13).

\section{Conclusion}

While hepatoid features are indeed rare and unusual morphological findings in urothelial carcinoma, it is important for pathologists to be aware of them in order to reach a correct diagnosis, particularly for a liver lesion when there is no other history of cancer, and the primary differential diagnosis may be hepatocellular carcinoma. Before a diagnosis of HAC or urothelial carcinoma with hepatoid features is made, a diagnosis of hepatocellular carcinoma must be excluded. Urothelial carcinoma with hepatoid features may exhibit an aggressive behavior, however, more information and studies with a large number of cases are needed to assess this possibility.

\section{References}

1 Humphrey PA, Moch H, Cubilla AL, Ulbright TM and Reuter VE: The 2016 WHO Classification of Tumours of the Urinary System and Male Genital Organs-Part B: Prostate and Bladder Tumours. Eur Urol 70: 106-119, 2016.

2 Chalasani V, Chin JL and Izawa JI: Histologic variants of urothelial bladder cancer and nonurothelial histology in bladder cancer. Can Urol Assoc J 3: S193-198, 2009.

3 Burgues O, Ferrer J, Navarro S, Ramos D, Botella E and Llombart-Bosch A: Hepatoid adenocarcinoma of the urinary bladder. An unusual neoplasm. Virchows Arch 435: 71-75, 1999.
4 Kawamura N, Hatano K, Kakuta Y, Takada T, Hara T and Yamaguchi S: A case of hepatoid adenocarcinoma of the urinary bladder. Hinyokika Kiyo 55: 619-622, 2009.

5 Lopez-Beltran A, Luque RJ, Quintero A, Requena MJ and Montironi R: Hepatoid adenocarcinoma of the urinary bladder. Virchows Arch 442: 381-387, 2003.

6 Sekino Y, Mochizuki H and Kuniyasu H: A 49-year-old woman presenting with hepatoid adenocarcinoma of the urinary bladder: a case report. J Med Case Rep 7: 12, 2013.

7 Sinard J, Macleay L Jr. and Melamed J: Hepatoid adenocarcinoma in the urinary bladder. Unusual localization of a newly recognized tumor type. Cancer 73: 1919-1925, 1994.

8 Yamada K, Fujioka Y, Ebihara Y, Kiriyama I, Suzuki H and Akimoto M: Alpha-fetoprotein producing undifferentiated carcinoma of the bladder. J Urol 152: 958-960, 1994.

9 Metzgeroth G, Strobel P, Baumbusch T, Reiter A and Hastka J: Hepatoid adenocarcinoma - review of the literature illustrated by a rare case originating in the peritoneal cavity. Onkologie 33 : 263-269, 2010.

10 Ishikura H, Ishiguro T, Enatsu C, Fujii H, Kakuta Y, Kanda M and Yoshiki T: Hepatoid adenocarcinoma of the renal pelvis producing alpha-fetoprotein of hepatic type and bile pigment. Cancer 67: 3051-3056, 1991.

11 Samaratunga H, Samaratunga D, Dunglison N, Perry-Keene J, Nicklin $J$ and Delahunt B: Alpha-fetoprotein-producing carcinoma of the renal pelvis exhibiting hepatoid and urothelial differentiation. Anticancer Res 32: 4987-4991, 2012.

12 Sakata Y, Onishi T, Yamada Y, Arima K, Yanagawa M and Kawamura J: alpha-Fetoprotein producing renal pelvic and ureter tumor. J Urol 166: 1830, 2001.

13 Monn MF, Kaimakliotis HZ, Pedrosa JA, Cary KC, Bihrle R, Cheng L and Koch MO: Contemporary bladder cancer: variant histology may be a significant driver of disease. Urol Oncol 33: 18.e15-20, 2015.

Received December 30, 2016

Revised January 16, 2017

Accepted January 17, 2017 